\title{
Mathematical Modeling of Crown Forest Fire Spread
}

\author{
Valeriy Perminov \\ Department of Mathematics and Natural Sciences, Belovo Branch of Kemerovo State University, Belovo, Russia \\ Email: p_valer@mail.ru
}

Received August 20 $0^{\text {th }}$, 2011; revised November 9 ${ }^{\text {th }}$, 2011; accepted November $17^{\text {th }}, 2011$

\begin{abstract}
Mathematical model of forest fire was based on an analysis of known experimental data and using concept and methods from reactive media mechanics. In this paper the assignment and theoretical investigations of the problems of crown forest fire spread in windy condition were carried out. In this context, a study — mathematical modeling — of the conditions of forest fire spreading that would make it possible to obtain a detailed picture of the change in the temperature and component concentration fields with time, and determine as well as the limiting condition of fire propagation in forest with fire break.
\end{abstract}

Keywords: Forest Fire; Mathematical Model; Turbulence; Ignition; Fire Spread; Control Volume; Discrete Analogue

\section{Introduction}

A great deal of work has been done on the theoretical problem of crown forest fire initiation. Crown fires are initiated by convective and radiative heat transfer from surface fires. However, convection is the main heat transfer mechanism (Van Wagner, 1977). The theory proposed by Van Wagner (1977) depends on three simple crown properties: crown base height, bulk density of forest combustible materials and moisture content of forest fuel. Also, crown fire initiation and hazard have been studied and modeled in details later (Alexander, 1998); Van Wagner (1989); Xanthopoulos (1990); Rothermel (1991); Cruz and others (2002); Albini and others (1195); Scott and Reinhardt (2001). The more complete discussion of the problem of crown forest fires is provided by coworkers at Tomsk University (Grishin, 1997); Grishin et al. (1998); Perminov (1995, 1998). In particular, a mathematical model of forest fires was obtained by Grishin (1997) based on an analysis of known and original experimental data; Konev (1977), and using concepts and methods from reactive media mechanics. The physical two-phase models used by Morvan \& Dupuy (2001, 2004) may be considered as a continuation and extension of the formulation proposed in (Grishin 1997). This study gives a two dimensional averaged mathematical setting and method of numerical solution of a problem of a forest fire spread. The boundary-value problem is solved numerically using the method of splitting according to physical processes. It was based on numerical solution of two dimensional Reynolds equations for the description of turbulent flow taking into account for diffusion equations chemical components and equations of energy conservation for gaseous and condensed phases, volume of fraction of condensed phase (dry organic substance, moisture, condensed pyrolysis products, mineral part of forest fuel).

\section{Mathematical Model}

It is assumed that the forest during a forest fire can be modeled as 1) a multi-phase, multistoried, spatially heterogeneous medium; 2) in the fire zone the forest is a porous-dispersed, two-temperature, single-velocity, reactive medium; 3 ) the forest canopy is supposed to be non-deformed medium (trunks, large branches, small twigs and needles), which affects only the magnitude of the force of resistance in the equation of conservation of momentum in the gas phase, i.e., the medium is assumed to be quasi-solid (almost non-deformable during wind gusts); 4) let there be a so-called "ventilated" forest massif, in which the volume of fractions of condensed forest fuel phases, consisting of dry organic matter, water in liquid state, solid pyrolysis products, and ash, can be neglected compared to the volume fraction of gas phase (components of air and gaseous pyrolysis products); 5) the flow has a developed turbulent nature and molecular transfer is neglected; 6) gaseous phase density doesn't depend on the pressure because of the low velocities of the flow in compareson with the velocity of the sound. Let the point $x_{1}, x_{2}, x_{3}=0$ is situated at the centre of the surface forest fire source at the height of the roughness level, axis $0 x_{1}$ directed parallel to the Earth's surface to the right in the direction of the unperturbed wind speed, axis $0 x_{2}$ directed perpendicular to $0 x_{1}$ and axis $0 x_{3}$ directed upward (Figure 1).

Because of the horizontal sizes of forest massif more than height of forest $-h$, system of equations of general mathematiccal model of forest fire (Grishin, 1997) was integrated between the limits from height of the roughness level -0 to $h$. Besides, suppose that

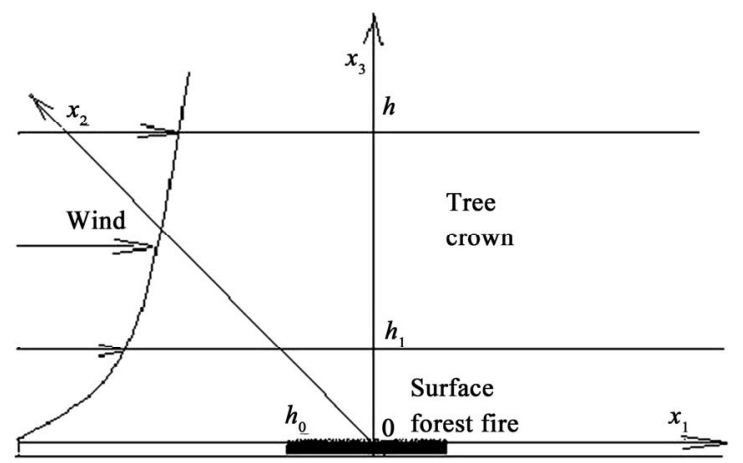

Figure 1.

Basic scheme of forest fire initiation and propagation. 


\section{PERMINOV}

$$
\int_{0}^{h} \phi \mathrm{d} x_{3}=\bar{\varphi} h
$$

$\dddot{\phi}$-average value of $\phi$. The problem formulated above is reduced to a solution of the following system of equation:

$$
\begin{aligned}
& \frac{\partial \rho}{\partial t}+\frac{\partial}{\partial x_{j}}\left(\rho v_{j}\right)=Q-\left(\dot{m}^{-}-\dot{m}^{+}\right) / h, \quad j=1,2,3 ; \\
& \rho \frac{\mathrm{d} v_{i}}{\mathrm{~d} t}=-\frac{\partial p}{\partial x_{j}}+\frac{\partial}{\partial x_{j}}\left(-\rho \vec{v}_{i}^{\prime} \vec{v}_{j}^{\prime}\right)-\rho s c_{d} v_{i}|\boldsymbol{v}|-\rho g_{i} \\
& -Q v_{i}+\left(\tau_{i}^{-}-\tau_{i}^{+}\right) / h, \quad i=1,2,3 ; \\
& \rho c_{p} \frac{\mathrm{d} T}{\mathrm{~d} t}=\frac{\partial}{\partial x_{j}}\left(-\rho c_{p} \overline{v_{j}^{\prime} T^{\prime}}\right)+q_{5} R_{5}-\alpha_{v}\left(T-T_{s}\right)+-\left(q_{T}^{-}-q_{T}^{+}\right) / h ; \\
& \rho \frac{\mathrm{d} c_{\alpha}}{\mathrm{d} t}=\frac{\partial}{\partial x_{j}}\left(-\rho \overline{v_{j}^{\prime} c_{\alpha}^{\prime}}\right)+R_{5 \alpha}-Q c_{\alpha}+\left(J_{\alpha}^{-}-J_{\alpha}^{+}\right) / h, \quad \alpha=\overline{1,5} \\
& \frac{\partial}{\partial x_{j}}\left(\frac{c}{3 k} \times \frac{\partial U_{R}}{\partial x_{j}}\right)-k\left(c U_{R}-4 \sigma T_{S}^{4}\right)+\left(q_{R}^{-}-q_{R}^{+}\right) / h=0 ; \\
& \sum_{i=1}^{4} \rho_{i} c_{p i} \phi_{i} \frac{\partial T_{S}}{\partial t}=q_{3} R_{3}-q_{2} R_{2}+k\left(c U_{R}-4 \sigma T_{S}^{4}\right)+\alpha_{V}\left(T-T_{S}\right) ; \\
& \rho_{1} \frac{\partial \varphi_{1}}{\partial t}=-R_{1}, \rho_{2} \frac{\partial \varphi_{2}}{\partial t}=-R_{2}, \\
& \rho_{3} \frac{\partial \varphi_{3}}{\partial t}=a_{C} R_{1}-\frac{M_{C}}{M_{1}} R_{3}, \rho_{4} \frac{\partial \varphi_{4}}{\partial t}=0 \\
& \sum_{a=1}^{5} c_{a}=1, p_{e}=\rho R T \sum_{a=1}^{5} \frac{c_{a}}{M_{a}}, \boldsymbol{v}=\left(v_{1}, v_{2}, v_{3}\right), \boldsymbol{g}=(0,0, g)
\end{aligned}
$$

The system of Equations (1)-(7) must be solved taking into account the initial and boundary conditions:

$$
\begin{aligned}
& t=1: v_{1}=0, v_{2}=0, v_{3}=0, T=T_{e}, c_{a}=c_{a e}, T_{s}=T_{e}, \varphi_{1}=\varphi_{i e} \\
& x_{1}=-x_{1 e}: v_{1}=V_{e}, v_{2}=0, \frac{\partial v_{3}}{\partial x_{1}}=0, T=T_{e}, c_{a}=c_{a e} \\
& -\frac{c}{3 k} \times \frac{\partial U_{R}}{\partial x_{1}}+c U_{R} / 2=0 \\
& x_{1}=x_{1 e}: \frac{\partial v_{1}}{\partial x_{1}}=0, \frac{\partial v_{2}}{\partial x_{1}}=0, \frac{\partial v_{3}}{\partial x_{1}}=0, \frac{\partial c_{\alpha}}{\partial x_{1}}=0, \frac{\partial T}{\partial x_{1}}=0 \\
& \frac{c}{3 k} \times \frac{\partial U_{R}}{\partial x_{1}}+\frac{c}{2} U_{R}=0 ; \\
& x_{2}=x_{20}: \frac{\partial v_{1}}{\partial x_{2}}=0, \frac{\partial v_{2}}{\partial x_{2}}=0, \frac{\partial v_{3}}{\partial x_{2}}=0, \frac{\partial c_{\alpha}}{\partial x_{2}}=0, \frac{\partial T}{\partial x_{2}}=0 \\
& -\frac{c}{3 k} \times \frac{\partial U_{R}}{\partial x_{2}}+\frac{c}{2} U_{R}=0 ; \\
& x_{2}=x_{2 e}: \frac{\partial v_{1}}{\partial x_{2}}=0, \frac{\partial v_{2}}{\partial x_{2}}=0, \frac{\partial v_{3}}{\partial x_{2}}=0, \frac{\partial c_{\alpha}}{\partial x_{2}}=0, \frac{\partial T}{\partial x_{2}}=0 \\
& \frac{c}{3 k} \times \frac{\partial U_{R}}{\partial x_{2}}+\frac{c}{2} U_{R}=0 ; \\
& x_{3}=0: v_{1}=0, v_{2}=0, \frac{\partial c_{\alpha}}{\partial x_{3}}=0,-\frac{c}{3 k} \frac{\partial U_{R}}{\partial x_{3}}+\frac{c}{2} U_{R}=0 \\
& v_{3}=v_{30}, T=T_{g},\left|x_{1}\right| \leq \Delta,\left|x_{2}\right| \leq \Delta \\
& v_{3}=0, T=T_{e},\left|x_{1}\right|>\Delta,\left|x_{2}\right|>\Delta ;
\end{aligned}
$$

$$
\begin{aligned}
x_{3}= & x_{3 e}: \frac{\partial v_{1}}{\partial x_{3}}=0, \frac{\partial v_{2}}{\partial x_{3}}=0, \frac{\partial v_{3}}{\partial x_{3}}=0, \frac{\partial c_{\alpha}}{\partial x_{3}}=0, \frac{\partial T}{\partial x_{3}}=0, \\
& \frac{c}{3 k} \times \frac{\partial U_{R}}{\partial x_{3}}+\frac{c}{2} U_{R}=0 .
\end{aligned}
$$

Here and above $\frac{\mathrm{d}}{\mathrm{d} t}$ is the symbol of the total (substantial) derivative; $\alpha v$ is the coefficient of phase exchange; $\rho$-density of gas-dispersed phase, $t$ is time; $v_{l}$-the velocity components; $T, T_{S}$-temperatures of gas and solid phases, $U_{R}$ - density of radiation energy, $k$-coefficient of radiation attenuation, $P$-pressure; $c_{p}$-constant pressure specific heat of the gas phase, $c_{p i}, \rho_{i}, \varphi_{1}$-specific heat, density and volume of fraction of condensed phase (1-dry organic substance, 2-moisture, 3condensed pyrolysis products, 4 -mineral part of forest fuel), $R_{l}$ - the mass rates of chemical reactions, $q_{i}$-hermal effects of chemical reactions; $k_{g}, k_{S}$-radiation absorption coefficients for gas and condensed phases; $T_{e}$-the ambient temperature; $c_{\alpha}$ mass concentrations of $\alpha$-component of gas-dispersed medium, index $\alpha=1,2$, 3 , where 1 corresponds to the density of oxygen, 2 -to carbon monoxide $\mathrm{CO}, 3$ - to carbon dioxide and inert components of air, 4, 5-soot and ash; $R$-universal gas constant; $M_{\alpha}, M_{C}$, and $M$ molecular mass of $\alpha$-components of the gas phase, carbon and air mixture; $g$ is the gravity acceleration; $c_{d}$ is an empirical coefficient of the resistance of the vegetation, $s$ is the specific surface of the forest fuel in the given forest stratum. In system of Equations (1)-(7) are introduced the next designations:

$$
\dot{m}=\rho v_{3}, \tau_{i}=-\rho \overline{v_{i}^{\prime} v_{3}^{\prime}}, J_{\alpha}=-\rho \overline{v_{3}^{\prime} c_{\alpha}^{\prime}}, J_{T}=-\rho \overline{v_{3}^{\prime} T^{\prime}}
$$

Upper indexes “+” and “-” designate values of functions at $x_{3}=h$ and $x_{3}=0$ correspondingly. It is assumed that heat and mass exchange of fire front and boundary layer of atmosphere are governed by Newton law and written using the formulas:

$$
\begin{aligned}
& \left(q_{T}^{-}-q_{T}^{+}\right) / h=-\alpha\left(T-T_{e}\right) / h, \\
& \left(J_{\alpha}^{-}-J_{\alpha}^{+}\right) / h=-\alpha\left(c-c_{\alpha e}\right) / h c_{p} .
\end{aligned}
$$

To define source terms which characterize inflow (outflow of mass) in a volume unit of the gas-dispersed phase, the following formulae were used for the rate of formulation of the gasdispersed mixture $\dot{m}$, outflow of oxygen $R_{51}$, changing carbon monoxide $R_{52}$.

$$
\begin{aligned}
& Q=\left(1-\alpha_{c}\right) R_{1}+R_{2}+\frac{M_{c}}{M_{1}} R_{3}, R_{51}=-R_{3}-\frac{M_{1}}{2 M_{2}} R_{5}, \\
& R_{52}=v_{g}\left(1-\alpha_{c}\right) R_{1}-R_{5}, R_{53}=0 .
\end{aligned}
$$

Here $v_{\mathrm{g}}$-mass fraction of gas combustible products of pyrolysis, $\alpha_{4}$ and $\alpha_{5}$-empirical constants. Reaction rates of these various contributions (pyrolysis, evaporation, combustion of coke and volatile combustible products of pyrolysis) are approximated by Arrhenius laws whose parameters (pre-exponential constant $k_{i}$ and activation energy $E_{i}$ ) are evaluated using data for mathematical models (Grishin, 1997; Perminov, 1995).

$$
\begin{aligned}
& R_{1}=k_{1} \rho_{1} \varphi_{1} \exp \left(-\frac{E_{1}}{R T_{s}}\right), R_{2}=k_{2} \rho_{2} \varphi_{2} T_{s}^{-0.5} \exp \left(-\frac{E_{2}}{R T_{s}}\right), \\
& R_{3}=k_{3} \rho \varphi_{3} S_{\sigma} c_{1} \exp \left(-\frac{E_{3}}{R T_{s}}\right), \\
& R_{5}=k_{5} M_{2}\left(\frac{c_{1} M}{M_{1}}\right)^{0.25} \frac{c_{2} M}{M_{2}} T^{-2.25} \exp \left(-\frac{E_{5}}{R T}\right) .
\end{aligned}
$$


The initial values for volume of fractions of condensed phases are determined using the expressions:

$$
\varphi_{1 e}=\frac{d\left(1-v_{z}\right)}{\rho_{1}}, \varphi_{2 e}=\frac{W d}{\rho_{2}}, \varphi_{3 e} \frac{a_{c} \varphi_{1 e} \rho_{1}}{\rho_{3}}
$$

where $d$-bulk density for surface layer, $v_{z}$-coefficient of ashes of forest fuel, $W$-forest fuel moisture content. It is supposed that the optical properties of a medium are independent of radiation wavelength (the assumption that the medium is "grey"), and the so-called diffusion approximation for radiation flux density were used for a mathematical description of radiation transport during forest fires.

To close the system (1)-(7), the components of the tensor of turbulent stresses, and the turbulent heat and mass fluxes are determined using the local-equilibrium model of turbulence (Grishin, 1997). The system of Equations (1)-(7) contains terms associated with turbulent diffusion, thermal conduction, and convection, and needs to be closed. The components of the tensor of turbulent stresses $\rho \overline{v^{\prime} w^{\prime}}$, as well as the turbulent fluxes of heat and mass $\overline{\rho v_{j}^{\prime} c_{p} T^{\prime}}, \overline{\rho v_{j}^{\prime} c_{a}^{\prime}}$ are written in terms of the gradients of the average flow properties using the formulas:

$$
\begin{aligned}
& -\rho \overline{v_{i}^{\prime} v_{j}^{\prime}}=\mu_{t}\left(\frac{\partial v_{i}}{\partial x_{j}}+\frac{\partial v_{j}}{\partial x_{i}}\right)-\frac{2}{3} K \delta_{i j}, \\
& -\rho \overline{v_{j} c_{p} T^{\prime}}=\lambda_{t} \times \frac{\partial T}{\partial x_{j}},-\rho \overline{v_{j} c_{a}^{\prime}}=\rho D_{t} \times \frac{\partial c_{a}}{\partial x_{j}} \\
& \lambda_{t}=\mu_{t} c_{p} / \operatorname{Pr}_{t}, \rho D_{t}=\mu_{t} / S c_{t}, \mu_{t}=c_{\mu} \rho K^{2} / \varepsilon,
\end{aligned}
$$

where $\mu_{t}, \lambda_{t}, D_{t}$ are the coefficients of turbulent viscosity, thermal conductivity, and diffusion, respectively; $\mathrm{Pr}_{t}, S c_{t}$ are the turbulent Prandtl and Schmidt numbers, which were assumed to be equal to 1 . In dimensional form, the coefficient of dynamic turbulent viscosity is determined using local equilibrium model of turbulence (Grishin, 1997). The system of Equations (1)-(7) must be solved taking into account the initial and boundary conditions. The thermodynamic, thermophysical and structural characteristics correspond to the forest fuels in the canopy of a different type of forest; such as, pine forest (Grishin, 1997).

\section{Numerical Methods and Results}

The boundary-value problem (1)-(13) is solved numerically using the method of splitting according to physical processes (Perminov, 1995). In the first stage, the hydrodynamic pattern of flow and distribution of scalar functions was calculated. The system of ordinary differential equations of chemical kinetics obtained as a result of splitting was then integrated. A discrete analog was obtained by means of the control volume method using the SIMPLE like algorithm (Patankar, 1981). The accuracy of the program was checked by the method of inserted analytical solutions. Analytical expressions for the unknown functions were substituted in (1)-(7) and the closure of the equations were calculated. This was then treated as the source in each equation. Next, with the aid of the algorithm described above, the values of the functions used were inferred with an accuracy of not less than $1 \%$. The effect of the dimensions of the control volumes on the solution was studied by diminishing them. The time step was selected automatically. Fields of temperature, velocity, component mass fractions, and volume fractions of phases were obtained numerically. The distribution of basic functions shows that the process of crown forest fire initiation goes through the next stages. The first stage is related to increasing maximum temperature in the fire source. At this process stage the fire source a thermal wind is formed a zone of heated forest fire pyrolysis products which are mixed with air, float up and penetrate into the crowns of trees. As a result, forest fuels in the tree crowns are heated, moisture evaporates and gaseous and dispersed pyrolysis products are generated. Ignition of gaseous pyrolysis products of the ground cover occurs at the next stage, and that of gaseous pyrolysis products in the forest canopy occurs at the last stage. As a result of heating of forest fuel elements of crown, moisture evaporates, and pyrolysis occurs accompanied by the release of gaseous products, which then ignite and burn away in the forest canopy. At the moment of ignition the gas combustible products of pyrolysis burns away, and the concentration of oxygen is rapidly reduced. The temperatures of both phases reach a maximum value at the point of ignition. The ignition processes is of a gas-phase nature. Note also that the transfer of energy from the fire source takes place due to radiation; the value of radiation heat flux density is small compared to that of the convective heat flux. At $V_{e} \neq 0$, the wind field in the forest canopy interacts with the gas-jet obstacle that forms from the forest fire source and from the ignited forest canopy and burn away in the forest canopy. Figures 2-5 present the distribution of temperature $\bar{T}\left(\bar{T}=T / T_{e}, T_{e}=300 \mathrm{~K}\right)(1-2,2-2.6,3-3,4-3.5,5$ 4) for gas phase, oxygen $\bar{c}_{1}(1-0.1,2$ - 0.5, 3 - 0.6, 4 - 0.7, 5 $0.8,6-0.9$ ), volatile combustible products of pyrolysis $\bar{c}_{2}$ concentrations (1 - 1, 2 - 0.1, 3 - 0.05, 4 - 0.01)

$\left(\bar{c}_{\alpha}=c_{\alpha} / c_{1 e}, c_{1 e}=0.23\right)$, temperature of condensed phase

$$
\bar{T}_{S}\left(\bar{T}_{S}=T_{S} / T_{e}, T_{e}=300 \mathrm{~K}\right)(1-2,2-2.6,3-3,4-3.5,5-4)
$$
for wind velocity $V e=10 \mathrm{~m} / \mathrm{s}$ at $h=10 \mathrm{~m}: 1) t=3 \mathrm{sec}$., 2) $t=$ $10 \mathrm{sec}$, 3) $t=18 \mathrm{sec}$., 4) $t=24 \mathrm{sec}$.

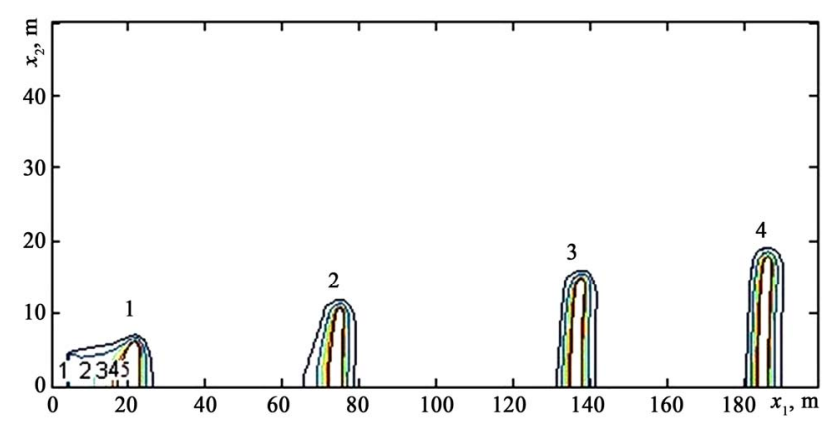

Figure 2.

Field of isotherms of the forest fire spread (gas phase).

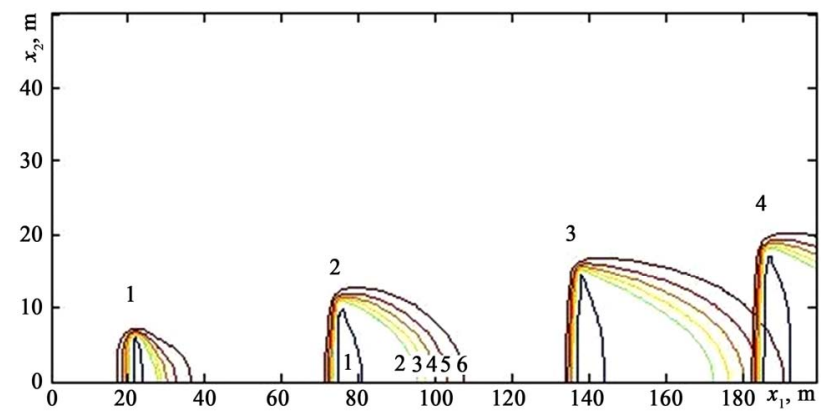

Figure 3.

The distribution of oxygen $\overline{c_{1}}$. 


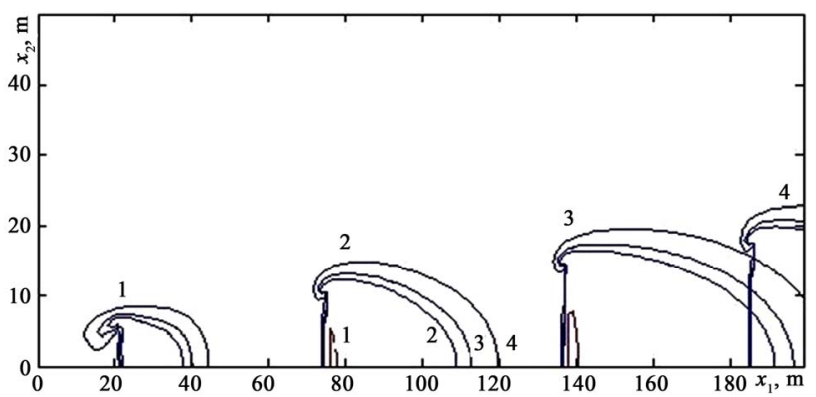

Figure 4.

The distribution of $\overline{c_{2}}$.

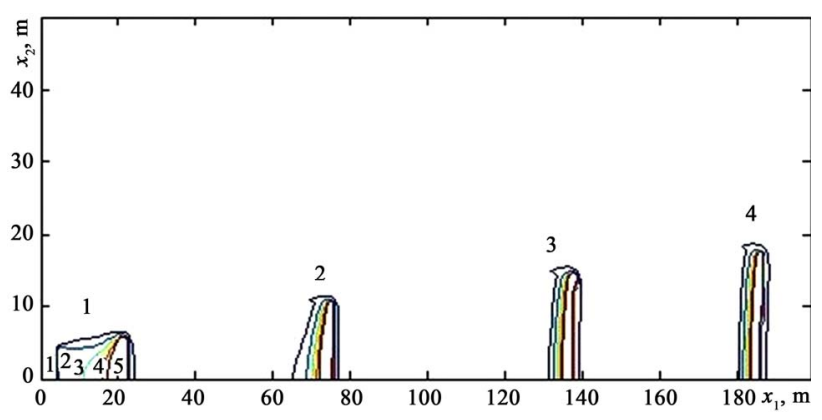

Figure 5.

Field of isotherms of the forest fire spread (solid phase).

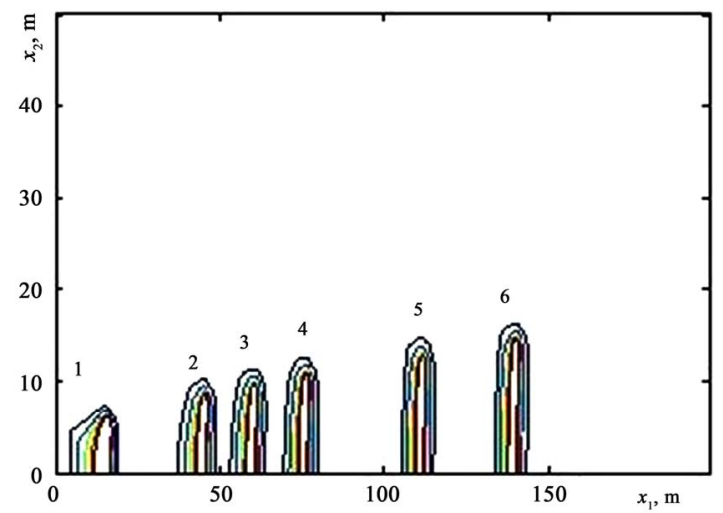

(a)

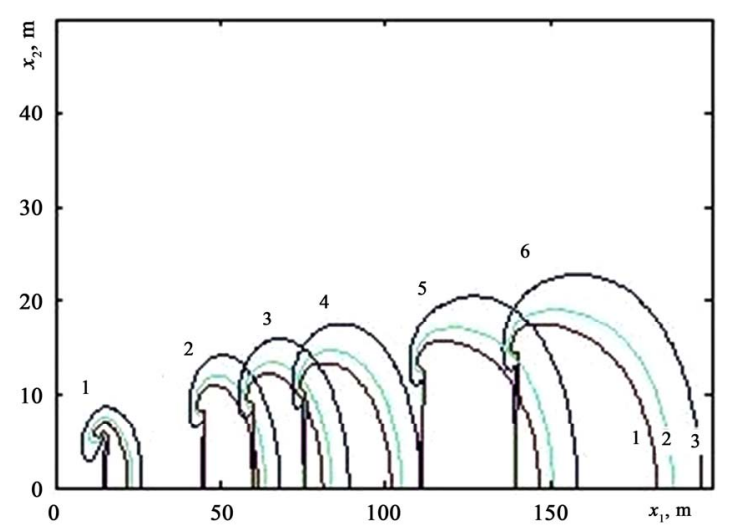

(c)
We can note that the isotherms and lines of equal levels are moved in the forest canopy and deformed by the action of wind. Similarly, the fields of component concentrations are deformed. It is concluded that the forest fire begins to spread. Mathematical model and the result of the calculation give an opportunity to consider forest fire spread for different wind velocity.

Figures 6(a)-(d) present the distribution of temperature for gas phase, concentration of oxygen and volatile combustible products of pyrolysis $c_{2}$ concentrations and temperature of condensed phase for wind velocity $V e=5 \mathrm{~m} / \mathrm{s}$ at $h=10 \mathrm{~m}: 1) t=3$ sec., 2) $t=10 \mathrm{sec}$., 3) $t=18 \mathrm{sec}$., 4) $t=20 \mathrm{sec}$., 5) $t=31 \mathrm{sec}$., 6) $t=40 \mathrm{sec}$. The results reported in Figure 6 show the decrease of the wind induces a decrease of the rate of fire spread.

One of the objectives of this paper could be to develop modeling means to reduce forest fire hazard in forest or near towns. In this paper it presents numerical results to study forest fire propagation through firebreak. This problem was considered by Zverev (1985) in one dimensional mathematical model approach.

Figures 7 and 8 (Figure 8(b) is a continuation of Figure 8(a)) present the forest fire front movement using distributions of temperature at different instants of time for two sizes of firebreaks ( 4.5 and 4 meters). The fire break is situated in the middle of domain $\left(x_{1}=100 \mathrm{~m}\right)$. In the first case the fire could not spread through this fire break.

If the fire break reduces to 4 meters (Figure 8) the fire continue to spread but the isotherm (isotherm 5) of forest fire is decreased after overcoming of fire break. In the Figure 9. The

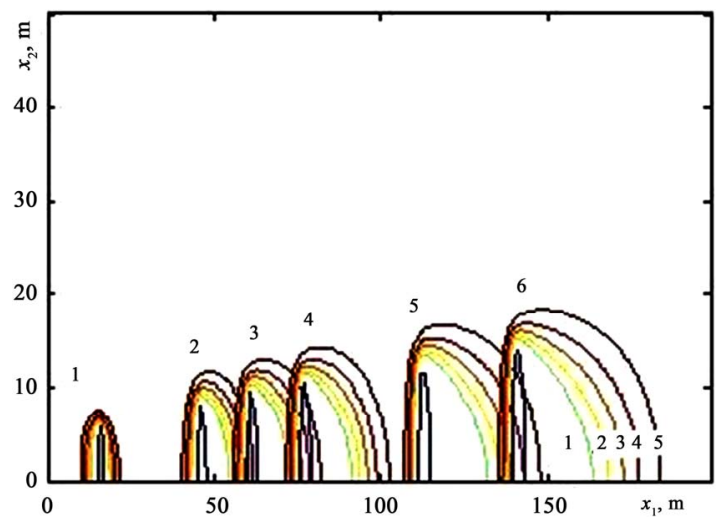

(b)

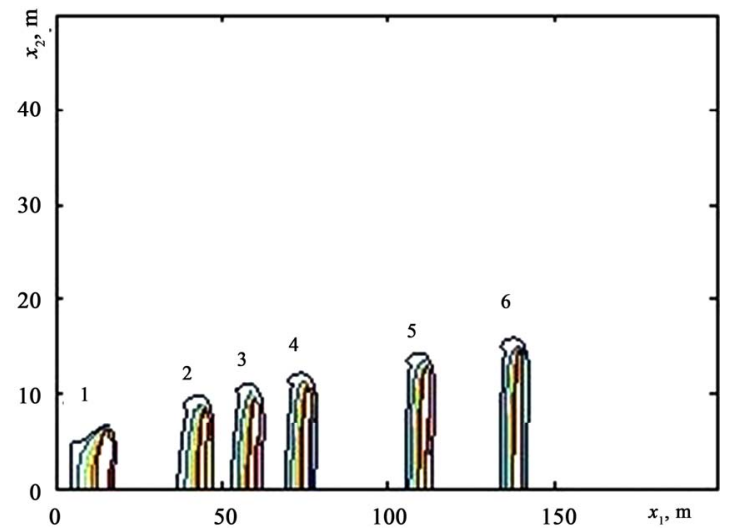

(d)

Figure 6.

Fields of isotherms of gas (a) and solid phase (d), isolines of oxygen (b) and gas products of pyrolysis (c). 


\section{PERMINOV}

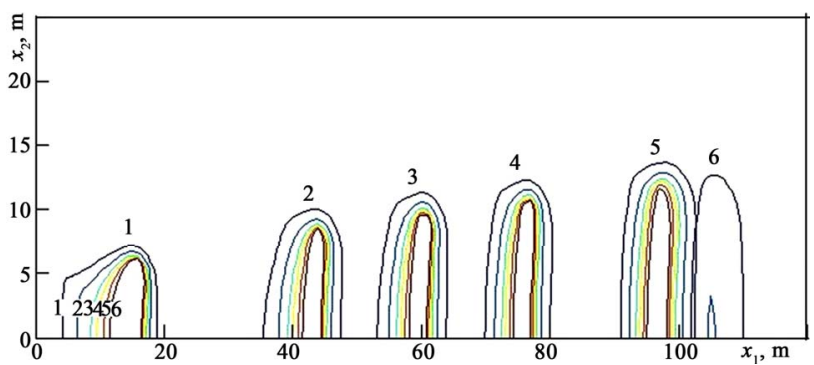

Figure 7.

Field of isotherms. Fire break equals $4.5 \mathrm{~m}$.

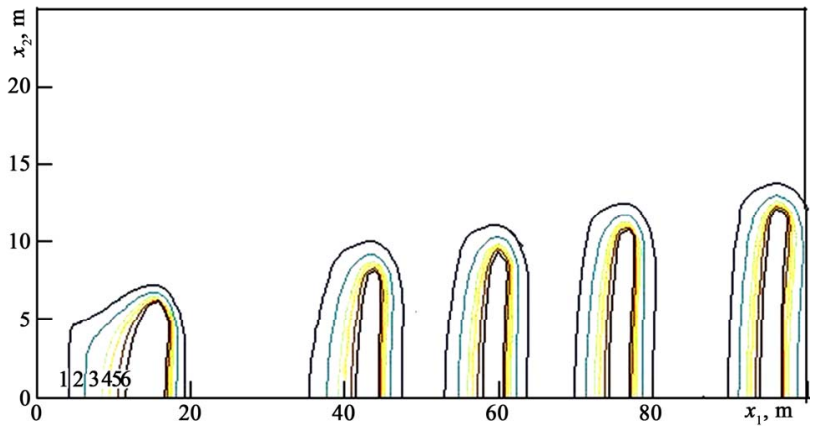

(a)

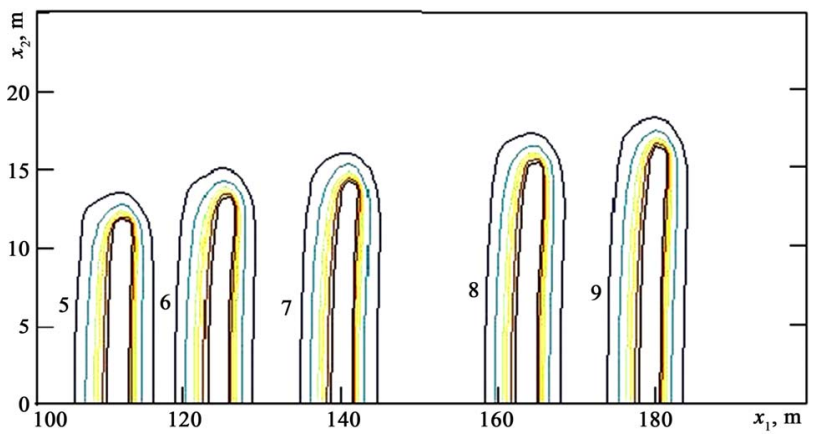

(b)

Figure 8.

Field of isotherms. Fire break equals $4 \mathrm{~m}$.

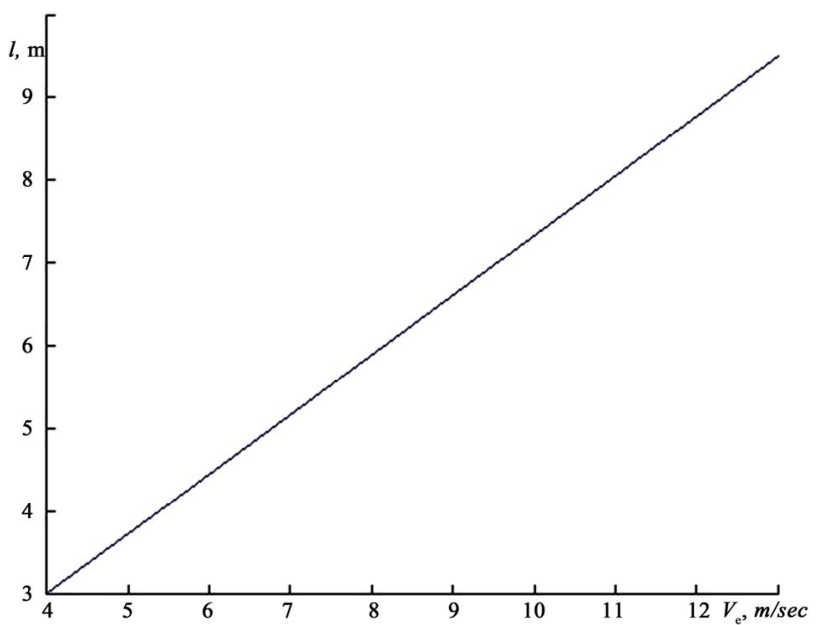

Figure 9.

The influence of wind velocity at the size of fire break. dependence of critical fire break value for different wind velocities is presented. Of course the size of safe distance depends not only of wind velocity, but type and quality of forest combustible materials, its moisture, height of trees and others conditions. This model allows to study an influence all these main factors.

\section{Conclusion}

The results of calculation give an opportunity to evaluate critical condition of the forest fire spread, which allows applying the given model for preventing fires. It overestimates the velocity of crown forest fire spread that depends on crown properties: bulk density, moisture content of forest fuel and etc. The model proposed there gives a detailed picture of the change in the temperature and component concentration fields with time, and determine as well as the influence of different conditions on the crown forest fire initiation. The results obtained agree with the laws of physics and experimental data (Konev, 1977; Grishin, 1997). From an analysis of calculations and experimental data it was found that for the cases in question the minimum total incendiary heat pulse is $2600 \mathrm{~kJ} / \mathrm{m}^{2}$ (Grishin, 1997). Calculations demonstrated that the value of the radiant heat flux for both problems is considerably less than the convective one, therefore radiation has a weak effect on local and integral characteristics of the problem discoursed above.

\section{REFERENCES}

Albini, F. A., et al. (1995). Modeling ignition and burning rate of large woody natural fuels. International Journal of Wildland Fire, 5, 81-91. doi:10.1071/WF9950081

Alexander, V. E. (1998). Crown fire thresholds in exotic pine plantations of Australasia. Ph.D. Thesis, Acton: Australian National University.

Cruz, M. G., Alexander, M. E., \& Wakimoto, R. H. (2002). Predicting crown fire behaviour to support forest fire management decision-making. In D. X. Viegas (Ed.), Proceedings of 4th international conference on Forest fire research/2002 wildland fire safety summit. Rotterdam: Millpress Science Publishers.

Grishin, A. M. (1997). Mathematical modeling forest fire and new methods fighting them. Tomsk: Publishing House of Tomsk University.

Grishin, A. M., \& Perminov, V. A. (1998). Mathematical modeling of the ignition of tree crowns. Combustion, Explosion, and Shock Waves, 34, 378-386. doi:10.1007/BF02675602

Konev, E. V. (1977). The physical foundation of vegetative materials combustion. Novosibirsk: Nauka.

Morvan, D., \& Dupuy, J. L. (2001). Modeling of fire spread through a forest fuel bed using a multiphase formulation. Combustion and Flame, 127, 1981-1994. doi:10.1016/S0010-2180(01)00302-9

Morvan, D., \& Dupuy, J. L. (2004). Modeling the propagation of wildfire through a Mediterranean shrub using a multiphase formulation. Combustion and Flame, 138, 199-210. doi:10.1016/j.combustflame.2004.05.001

Patankar, S. V. (1981). Numerical heat transfer and fluid flow. New York: Hemisphere Publishing Corporation.

Perminov, V. A. (1995). Mathematical modeling of crown and mass forest fires initiation with the allowance for the radiative-Convective heat and mass transfer and two temperatures of medium. Ph.D Thesis, Tomsk: Tomsk State University.

Perminov, V. A. (1998). Mathematical modeling of crown forest fire initiation. Proceedings of III International conference on forest fire research and 14th conference on fire and forest meteorology. Luso.

Rothermel, R. C. (1991). Crown fire analysis and interpretation. Proceedings of 11th international conference on Fire and forest meteor- 


\section{PERMINOV}

ology. Bethesda, MA: Society of American Foresters.

Scott, J. H., et al. (2001). Assessing crown fire potential by linking models of surface and crown fire behavior. USDA Forest Service, Rocky Mountain Forest and Range Experiment Station. Colorado: Fort Collins.

Van Wagner, C. E. (1977). Conditions for the start and spread of crown fire. Canadian Journal of Forest Research, 7, 23-34. doi:10.1139/x77-004
Van Wagner, C. E. (1989). Prediction of crown fire behavior in conifer stands. Proceedings of 10th conference on fire and forest meteorology. Ottawa, Ontario: Forestry Canada.

Xanthopoulos, G. (1990). Development of a wildland crown fire initiation model. Ph.D. Thesis, Missoula: University of Montana.

Zverev, V. G. (1985). Mathematical modeling of aerodynamics and heat and mass transfer at crown forest fire spread. Ph.D. Thesis, Tomsk: Tomsk State University. 